\title{
Knee Contact Forces are Not Altered in Early Knee Osteoarthritis
}

Authors: S. Meireles ${ }^{1}$, ME; F. De Groote, $\mathrm{PhD}^{2}$; N. D. Reeves ${ }^{3}, \mathrm{PhD} ; \mathrm{S}$. Verschueren ${ }^{4}, \mathrm{PhD}$;

C. Maganaris ${ }^{5}, \mathrm{PhD} ; \mathrm{F}$. Luyten ${ }^{6}, \mathrm{PhD} ; \mathrm{I}$. Jonkers ${ }^{1}, \mathrm{PhD}$

${ }^{1}$ Department of Kinesiology, KU Leuven, Belgium

${ }^{2}$ Department of Mechanical Engineering, KU Leuven, Belgium

3 School of Healthcare Science, Manchester Metropolitan University, United Kingdom

${ }^{4}$ Department of Rehabilitation Sciences, KU Leuven, Belgium

${ }^{5}$ School of Sport and Exercise Sciences, Liverpool John Moores University, United Kingdom

${ }^{6}$ Department of Development and Regeneration, KU Leuven, Belgium

susana.meireles@faber.kuleuven.be,Friedl.DeGroote@mech.kuleuven.be,

N.Reeves@mmu.ac.uk, Sabine.Verschueren@faber.kuleuven.be, c.maganaris@1jmu.ac.uk, frank.luyten@uz.kuleuven.ac.be,ilse.jonkers@faber.kuleuven.be

Author for correspondence:

Susana Meireles

Tervuursevest 101

Gebouw De Nayer, lokaal

B-3001 Leuven - Heverlee

Phone: +3216379463

E-mail: susana.meireles@faber.kuleuven.be

Running Head: External moments vs internal KCF in OA 


\section{Introduction}

Osteoarthritis $(\mathrm{OA})$ is a chronic degenerative and multifactorial $(1,2)$ joint disease that most frequently affects the knee (3). Patients complain about pain, reduced range of joint movement, muscle weakness, stiffness and instability, which limits physical activities in daily living (4), results in loss of their independence, reduced quality of life and high health-related $\operatorname{costs}(5)$.

The cause of OA remains unclear. It is known that biochemical and mechanical factors may contribute to its initiation (6-9). Indeed, subchondral bone remodeling (10) following mechanical overloading will increase the reactive stresses underneath the cartilage, therefore decreasing the shock absorbing efficiency of cartilage (11) and causing local cartilage lesions (12). In agreement with this statement, aberrant knee joint loading has been identified as a factor affecting the progression of knee OA (13-15) in more advanced stages of OA (16): increased medial compartment loading has been associated with more pronounced clinical symptoms and OA severity as assessed by radiography $(24,25)$. Most studies (17-24) used the knee adduction moments (KAM), i.e. the external knee joint moment in the frontal plane was used as an indirect measure of medial compartment loading during functional activities. Alternatively, musculoskeletal modeling in combination with dynamic motions has been used to calculate knee contact forces. Using this approach, Kumar et al. (26) found medial KCF were increased in established OA subjects $(K \& L \geq 2)$ with radiographic signs of joint structural changes. Interestingly, medial compartment loading of the knee was found to be related to a combination of both KAM and knee flexion moment (KFM), therefore questioning the role of KAM as sole indicator of medial compartment knee loading.

More recently, clinical interest is towards identifying OA patients in more early stages of the disease process. Early detection of OA may enable more effective interventions before major 
structural damage has occurred (29). The lack of effectiveness in delaying the progression of OA (30) may be mainly caused by a late intervention, when structural deterioration is already advanced (31). Luyten et al. (27) have proposed a classification criteria for identifying early knee OA patients, which combines knee pain and Kellgren and Lawrence (K\&L) radiographic classification (0 or 1) (28) with structural changes detected on Magnetic Resonance Imaging (MRI) or cartilage lesions by arthroscopy.

The role of mechanical loading in these patients where only early signs of joint degeneration are present, is less well explored in literature. Three recent articles have shown that there is no evidence of increased KAM in early stages of knee OA compared to healthy controls $(16,25,32)$. However, since KAM does not account fully for the internal knee joint loading $(26,33)$, these studies potentially fail to describe the more subtle changes in loading characteristics in the early OA patients where structural degeneration is less pronounced.

The current study is therefore the first study to evaluate whether knee loading as assessed by $\mathrm{KCF}$, is different in subjects with early medial knee OA compared to healthy subjects and subjects with established medial knee OA. It is hypothesized that in the presence of early signs of structural degeneration as present in early OA subjects, knee loading is increased compared to healthy subjects but to a lesser extent than in established OA subjects. If so, this would confirm that biomechanical overloading is a contributing factor to the progression of OA from the very early onset of the disease. Furthermore, if subjects with early OA present increased knee loading will confirm KCF to be a more sensitive biomarker than KAM in detecting alterations in knee loading in early stages of OA, allowing evaluation of treatment effect even in early stages of the disease process and allowing for earlier interventions.

Furthermore, this study evaluates the contribution of altered frontal and sagittal plane moments to the observed changes in knee loading for subjects in different stages of the 
disease process. It is hypothesized that in early OA patients, presenting limited structural degeneration, frontal plane moments will contribute less to the observed changes in knee loading compared to established OA. If so, alterations in mechanical knee loading, associated with different levels of joint degeneration, relate to alterations in multidimensional joint loading, with KAM being a more important contributor compared to KFM in patients with established knee OA will be confirmed. 


\section{Methods}

\section{Participants}

Fifty-nine participants (all women, mean age of 65 years) were recruited for this study and were separated into three groups based on a previously published classification (27): control subjects $(n=20)$, early medial knee OA $(n=16)$, and established medial knee OA $(n=23)$ patients. Subject characteristics are listed in Table 1. All procedures were approved by the local ethical committee of Biomedical Science, KU Leuven, Belgium. Written informed consent was obtained from each subject.

Early medial knee OA was diagnosed based on novel classification criteria of Luyten et al. (27), including fulfillment of three criteria, namely knee pain, a K\&L (28) grade 0,1 or $2^{-}$ (osteophytes only) and structural changes observed on MRI.

Established medial knee OA was diagnosed based on slightly adapted American College of Rheumatology classification criteria (34), including knee pain, stiffness less than 30 min and crepitus, together with structural changes defined as presence of minimum grade $2^{+}$ (osteophytes and joint space narrowing) on $\mathrm{K} \& \mathrm{~L}$ scale for at least the medial compartment on radiography.

A control group was also analyzed, which included asymptomatic healthy subjects with no history of knee OA or other pathology involving any lower extremity joints, and with a radiological score of 0 or 1 according to K\&L score.

Participants were excluded if they had a prior significant trauma or surgery in lower limbs and/or low back, if they suffered from a neurological disease affecting coordination and/or balance during gait and/or a musculoskeletal disorders other than knee OA in one of the limbs during the last six months prior to testing. 
For symptomatic patients with unilateral knee OA $(n=9)$, only data of the affected knee were analyzed. For those with bilateral knee OA and with large asymmetry in severity $(n=7)$, the most affected side was selected for further analysis. For all other subjects $(n=23)$, both legs were analyzed.

\section{Gait analysis}

An active 3D motion analysis system (Krypton, Metris) recorded the 3D position of 27 LEDs attached to the subjects according to an extended (5 technical clusters and 12 LEDs on 6 anatomical landmarks) Helen Hayes protocol at a sampling frequency of $100 \mathrm{~Hz}$ (Figure 1 Supplementary Material).

A force plate (Bertec Corporation, USA), embedded in the middle of the walkway, measured ground reaction forces sampled at $1000 \mathrm{~Hz}$.

Gait analysis consisted of level walking along a $10 \mathrm{~m}$ walkway at self-selected speed. A total of 12 stance trials were averaged for controls and also for patients with bilateral OA with similar severity classification for both legs and 6 for the patients who had a less/no affected leg. Barefoot walking was chosen in order to optimize standardization since variation in footwear would influence lower limb loads (35).

Marker data were labeled and smoothed using a spline routine (36) in Matlab (Mathworks, inc.). The remainder of the analysis was performed using the standard workflow in Opensim (37): the 3D musculoskeletal model of the lower body (38) was extended with a 2 degrees of freedom knee joint: flexion/extension and adduction/abduction. First, the model was scaled based on the marker positions and the subject's body mass. Thereafter, joint angles were calculated by inverse kinematics. Joint reaction forces and moments were obtained by inverse dynamics. Knee joint moments were normalized to body weight and height $(\% \mathrm{BWHt}$, 
$\mathrm{N} / \mathrm{kg} \mathrm{ms}^{-2}$ ). A static optimization routine (39) that minimizes the sum of muscle activation squared was used to calculate individual muscle forces. Finally, KCF, resulting from the muscle forces and resultant forces were calculated during stance phase. KCF are expressed relative to the tibia reference frame with the origin in the knee joint center and normalized to body weight (BW, N/kg.s $\mathrm{s}^{-2}$. All data were time normalized to stance phase, from initial contact (heel strike) to toe-off.

\section{Data analysis}

Maximal total KCF, KAM and KFM during the first and second half of the stance phase and minimum values of the same parameters during the single support (SS) phase were determined.

Given the decreased walking speed (40) and concomitant prolonged stance phase $(41,42)$ present in patients with OA, the KAM and KFM angular impulse and $\mathrm{KCF}$ impulse were also analyzed. These correspond to the time integral of the moments and the total KCF and account for changes in both load magnitude and duration.

\section{Statistical analysis}

One-way analysis of variance (ANOVA) with Gabriel post hoc test (SPSS Inc., v17.0) evaluated whether differences in peaks KCF, KAM and KFM as well as their impulses were significantly different $(\mathrm{p} \leq 0.05)$.

To investigate the contribution of KAM and KFM to the KCF, values of KAM and KFM at the three time instants of peaks and SS were correlated to KCF. First, coefficient of determination $\left(\mathrm{R}^{2}\right)$ between KAM and $\mathrm{KCF}$, and between KFM and KCF was calculated in order to assess how much variance in KCF was explained by KAM and KFM, respectively. Multiple regression was then calculated to assess how much variation in KCF was explained 
by the combination of KAM and KFM. A linear relationship was assumed between KAM and $\mathrm{KCF}, \mathrm{KFM}$ and $\mathrm{KCF}$, and, finally, between $\mathrm{KAM}$ together with $\mathrm{KFM}$ and $\mathrm{KCF}$. Multicollinearity between KAM and KFM was verified for peaks and SS by the variance inflation factor (VIF) and tolerance $(\mathrm{T})$ values $(43,44)$ and was found to be negligible (Table 1 - Supplementary Material). 


\section{Results}

\section{Subject characteristics}

Age, body mass, gait speed, stance duration and timing of the peak KCF did not differ significantly between the three groups (Table 1). Single support phase was significantly shorter in patients with established OA compared to control subjects $(p=0.040)$. Significantly higher varus alignment was observed in patients with established OA compared to the control group $(p=0.022)$.

\section{Knee joint loading}

First peak KAM was significantly different between groups $(p=0.038)$. However, although higher KAM were observed in established OA patients (Figure 1), no significant differences were found when pairwise comparisons were done. KFM peaks were not significantly different between any of the three groups. In contrast, significant lower KFM ( $p=0.013)$ was found during SS in early OA when compared to established OA.

$\mathrm{KCF}$ is highest during the first peak, in all patient groups, particularly in patients with established OA. However, no statistically significant differences were found between the groups in terms of first and second peak loading (KCF) (Table 2). During midstance (SS), the early OA group showed significantly lower KCF compared to established OA $(p=0.022)$.

KAM and KFM angular impulses did not significantly differ between groups (Table 3). However, KCF impulses were significantly increased in established OA subjects when compared to control group ( $p=0.033)$ and early OA $(p=0.018)$. 
During first peak and SS, KAM correlates significantly to $\mathrm{KCF}(p<0.01)$ in both patient groups, with the highest contribution in the established OA subjects (up to $74 \%$, Table 4). Although lower contributions were found for KFM compared to KAM, the contributions of KFM were higher in the OA groups compared to controls with the highest contribution in early OA subjects (up to 62\%). The combination of KAM and KFM better predicted KCF, increasing the prediction up to $91 \%$ and $95 \%$ in the patient groups.

During the second peak KCF, KAM did not predict $\mathrm{KCF}$ in both patient groups. KFM contributed only significantly $(p<0.01)$ to KCF in the early OA group (variance predicted $55 \%$ ). In early OA, the variance of the KCF accounted for when combining KFM and KAM was similar to that in control subjects. In contrast, in patients with established OA, the variance in KCF explained when combining KAM and KFM remained very low (20\%). 


\section{Discussion}

This study investigated mechanical knee loading in terms of external moments (KFM and KAM) as well as knee contact forces (KCF) during gait in subjects with early knee OA compared to controls and established knee OA using musculoskeletal modeling and dynamic simulations of motion. We aimed to investigate the presence of altered knee joint loading in early knee OA where structural degeneration is limited compared to established OA as well as the extent to which alterations in the frontal and sagittal plane moments contribute to the observed changes in knee loading.

Mechanical loading was not significantly higher in early OA subjects compared to controls, not when considering the external moments (KAM or KFM), nor knee contact forces (KCF). This finding falsifies the first hypothesis. From this we conclude that no signs of increased knee loading are present in subjects that only present early signs of structural joint degeneration. These findings are in line with Baert et al. (25) and Duffell et al. (32), who found no differences in KAM between early OA and healthy subjects. Therefore, the potential use of knee contact forces during walking to detect treatment effect on early OA was not confirmed. However, it is important to recognize that only walking has been evaluated in this study and that this may not be representative for an overall functional status of the subjects. Indeed, Hensor et al. (45) reported knee pain first during weight-bearing activities involving deep knee bending, such as climbing or descending stairs, since they are more challenging. Future research should therefore focus on studying knee loading during these more demanding tasks as they may be more sensitive in detecting early changes in knee loading in OA subjects. 
Mechanical loading was higher in established OA compared to early OA subjects. Indications for higher knee loading were statistically confirmed for increased knee contact force impulses. These were significantly higher in the established compared to the early OA subjects, representative for the cumulative effect of increased loading magnitude and prolonged stance duration in the established OA group. It is important to note that in the current groups, increased loading was not statistically confirmed when only considering the peak knee contact forces or the peak external joint moments. The tendency of increased KAM and KCF is in line with the results of Baert, Kumar et al. (26) and Richards et al (46). However, loading during single stance was significantly increased in established OA as reflected in the higher KCF and KFM during single stance. These findings are in line with the reported changes in KFM during single stance reported in the study of Baert et al. (25). These findings partially confirm the first hypothesis and further support the presence of increased loading in later stages of OA where more structural joint degeneration is present.

$\mathrm{KCF}$ relates to the multidimensional contribution of the external moments of the knee joint. A good prediction of the variance in KCF during the first peak, where the knee contact force magnitude is maximal, is found for all groups when considering KAM and KFM. Although during initial double support, knee loading is predicted well by KAM irrespective the presence of OA, multiple regression results show that a combination of KAM and KFM leads to a better prediction of KCF than KAM or KFM alone, which is in agreement with previous studies $(26,47)$. Therefore, in agreement with our second hypothesis, we can conclude that both frontal and sagittal plane moments need to be considered to estimate KCF.

However, in established OA patients, the variance accounted for when combining KAM and KFM is low (20\%) during second part of the stance phase. This highlights the important role of muscle action controlling flexion-extension and adduction-abduction moments in joint loading during late stance. 
With increased structural joint degeneration, peak mechanical knee loading is differently influenced by the frontal and sagittal knee moments. When initial structural degeneration is present, KFM contributes more to the KCF. When structural degeneration increases, the contribution of KAM increases. Except for the second half of stance, where KAM could not predict the peak KCF.

\section{Limitations of this study}

These results have to be interpreted in view of certain methodological limitations. Ligaments were not included, assuming that external moments are generated entirely by the muscletendon structures. For that reason, the $\mathrm{KCF}$ is calculated without differentiating between medial and lateral compartment. In the current approach, the same control strategy (minimal effort) for controls and OA patients was assumed. In future research, passive and ligamentous structures will be incorporated in EMG-constrained muscle force computation.

\section{Conclusions}

Based on the followed modeling approach, excessive mechanical loading is not present during gait in early stages of OA but only in established OA compared to controls. This suggests excessive loading is not a contributor to early progression of OA, but may only result after later structural degeneration. Furthermore, KFM was essential to estimate KCF during the second peak in early OA. Therefore, KAM combined with KFM (rather than KAM on its own) is necessary to better estimate $\mathrm{KCF}$ and therefore might be used as feedback signal during gait retraining sessions aimed at assessing knee loading in patients with knee osteoarthritis. However, caution is required when assessing changes in KCF from changes only at the level of external moments in established OA patients, especially during the second half of the stance. 


\section{Author contributions}

All authors take responsibility for the integrity of the work as a whole, including data and accuracy of the analysis. Conception: S. Meireles, F. De Groote, ND. Reeves, I. Jonkers. Design: S. Verschueren. All the authors contributed to the analysis and interpretation of the data, drafting of the article and final approval of the article.

\section{Funding/Acknowledgements}

Integrated in Move-Age program funded by the European Commission as part of the Erasmus Mundus program.

\section{Competing interests}

All authors declare no conflict of interest. 


\section{References}

1. Lories RJ, Luyten FP. The bone-cartilage unit in osteoarthritis. Nat Rev Rheumatol 2011; 7(1):43-49.

2. Andriacchi TP, Mundermann A, Smith RL, Alexander EJ, Dyrby CO, Koo S. A framework for the in vivo pathomechanics of osteoarthritis at the knee. Ann Biomed Eng $2004 ; 32(3): 447-457$.

3. Buckwalter JA, Martin JA. Osteoarthritis. Adv Drug Deliv Rev 2006; 58(2):150-67.

4. Bhatia D, Bejarano T, Novo M. Current interventions in the management of knee osteoarthritis. J Pharmacy \& Bioallied Sciences 2013;5(1):30-38.

5. Fitzgerald GK, Piva SR and Irrgang JJ. Reports of joint instability in knee osteoarthritis: Its prevalence and relationship to physical function. Arthritis Rheum 2004; 51: 941-946.

6. Brandt KD, Doherty M, Lohmander LS. Introduction: the concept of ostearthritis as failure of the diarthrodial joint. In: Brandt KD, Doherty M, Lohmander LS (eds). Osteoarthritis, 2nd ed.. New York: Oxford University; 2003. p. 69-71.

7. Goldring MB and Goldring SR. Osteoarthritis. J Cell Physiol, 2007; 213(3): 626-34.

8. Liikavaino T. Biomechanics of gait and physical function in patients with knee osteoarthritis: thigh muscle properties and joint loading assessment. Publ. Univ. Eastern Finland. Dissertations in Health Sciences 2010; 13:98.

9. Radin EL, and Rose RM. Role of subchondral bone in the initiation and progression of cartilage damage. Clin Orthop Rel Res 1986; 34-40.

10. Burr DB. The importance of subchrondal bone in the progression of osteoarthritis, $\mathrm{J}$ Rheumatol. Sup 2004; 70: 77-80. 
11. Runhaar J, Koes BW, Clockaerts S, Bierma-Zeinstra SM. A systematic review on changed biomechanics of lower extremities in obese individuals: a possible role in development of osteoarthritis. Obes Rev 2011;12:1071-82.

12. Henriksen M. The significance of pain in knee joint loading during walking, Aalborg: Center for Sensory-Motor Interaction (SMI), Department of Health Science and Technology, Aalborg University 2007.

13. Nuki G, Salter D. The impact of mechanical stress on the pathophysiology of osteoarthritis. In: Sharm K \& Berenbaum F (eds). Osteoarthritis. Philadelphia: Mosby 2007; p. 33-52.

14. Sharma L. Local factors in osteoarthritis. Curr Opin Rheumatol 2001; 13(5): 441-6.

15. Brouwer GM, van Tol AW, Bergink AP, Belo JN, Bernsen RM, Reijman M, et al. Association between valgus and varus alignment and the development and progression of radiographic osteoarthritis of the knee. Arthritis Rheum 2007; 56(4): 1204-11.

16. Foroughi N, Smith R, Vanwanseele B. The association of external knee adduction moment with biomechanical variables in osteoarthritis: a systematic review. Knee 2009; 16, 303-309.

17. Lewek M, Rudolph KS, Snyder-Mackler L. Control of frontal plane knee laxity during gait in patients with medial compartment knee osteoarthritis. Osteoarthr Cartil 2004; 12(9):745-51.

18. Andriacchi T. Dynamics of knee malalignment. Orthop Clin North Am 1994; 25:395.

19. Fregly BJ, Reinbolt JA, Rooney KL, Mitchell KH, Chmielewski TL. Design of patient-specific gait modifications for knee osteoarthritis rehabilitation. IEEE Trans Biomed Eng 2007; 54:1687-1695.

20. Mundermann A, Asay JL, Mundermann L, Andriacchi. Implications of increased medio-lateral trunk sway for ambulatory mechanics. J Biomech 2008; 41:165-170. 
21. Hurwitz DE, Ryals AR, Block JA, Sharma L, Schnitzer TJ, Andriacchi TP. Knee pain and joint loading in subjects with osteoarthritis of the knee. J Orthop Res 2000; 18:572-579.

22. Guo M, Axe MJ, Manal K. The influence of foot progression angle on the knee adduction moment during walking and stair climbing in pain free individuals with knee osteoarthritis. Gait Posture 2007; 26:436-441.

23. Miyazaki T, Wada M, Kawahara H, Sato M, Baba H, Shimada S. Dynamic load at baseline can predict radiographic disease progression in medial compartment knee osteoarthritis. Ann Rheum Dis 2002; 61:617-622.

24. Baliunas A, Hurwitz D, Ryals A, Karrar A, Case JP, Block JA, Andriacchi TP. Increased knee joint loads during walking are present in subjects with knee osteoarthritis. Osteoarthr Cartil 2002; 10:573-579.

25. Baert IA, Jonkers I, Staes F, Luyten FP, Truijen S, Verschueren S. Gait characteristics and lower limb muscle strength in women with early and established knee osteoarthritis. Clin Biomech (Bristol, Avon) 2013; 28(1):40-7.

26. Kumar D, Manal KT, Ridolph KS. Knee joint loading during gait in healthy controls and individuals with knee osteoarthritis. Osteoarthr Cartil 2013; 21, 298-305.

27. Luyten FP, Denti M, Filardo G, Kon E, Engebretsen L. Definition and classification of early osteoarthritis of the knee. Knee Surg. Sports Traumatol Arthrosc 2012; 20, 401-406. 28. Kellgren JH, Lawrence JS. Radiological assessment of osteoarthrosis. Ann Rheum Dis., $1957 ; 16: 494-502$.

29. Guermazi A, Niu J, Hayashi D, Roemer FW, Englund M, Neogi T, et al. Prevalence of abnormalities in knees detected by MRI in adults without knee osteoarthritis: population based observational study. BMJ, 2012.

30. McAllindon TE, Bannuru RR, Sullivan MC, Arden NK, Berenbaum F, BiermaZeinstra SM, et al. OARSI guidelines for the non-surgical management of knee osteoarthritis. Osteoarthritis Cartilage 2014, 22:363-88. 
31. Felson DT, Hodgson R. Identifying and treating preclinical and early osteoarthritis. Rheum Dis Clin North Am 2014;40:699-710.

32. Duffell, LD, Southgate DFL, Gulati, V, McGregor, AH. Balance and gait adaptations in patients with early knee Osteoarthritis, Gait \& Posture, 39, 2014, pp. 1057-61.

33. Meyer AJ, D'Lima DD, Besier TF, Lloyd DG, Colwell CW, Fregly BJ. Are external knee load and EMG measures accurate indicators of internal knee contact forces during gait?. J Orthop Res 2013; 31(6); 921-9.

34. Altman R, Asch E, Bloch D, Bole G, Borenstein D, Brandt K, et al. Development of criteria for the classification and reporting of osteoarthritis. Classification of osteoarthritis of the knee. Diagnostic and Therapeutic Criteria Committee of the American Rheumatism Association. Arthritis Rheum 1986; 29:1039-1049.

35. Shakoor N, Block JA. Walking barefoot decreases loading on the lower extremity joints in knee osteoarthritis. Arthritis Rheum 2006; 54:2923-2927.

36. Woltring HJ. A Fortran package for generalized, cross-validatory spline smoothing and differentiation. Adv Eng Softw 1986; 8:104-113.

37. Delp SL, Anderson FC, Arnold AS, Loan P, Habib A, John CT, Guendelman E, Thelen DG. OpenSim: open-source software to create and analyze dynamic simulations of movement. IEEE Trans Biomed Eng 2007; 54:1940-1950.

38. Delp SL, Loan JP, Hoy MG, Zajac FE, Topp EL, Rosen JM. An interactive graphicsbased model of the lower extremity to study orthopaedic surgical procedures. IEEE Trans Biomed Eng 1990; 37:757-767.

39. Anderson FC and Pandy MG. Static and dynamic optimization solutions for gait are practically equivalent. J Biomech 2001; 34:153-161.

40. Kaufman KR, Hughes C, Morrey BF, Morrey M, An KN. Gait characteristics of patients with knee osteoarthritis. J Biomech 2001;34:907-15. 
41. Al Zahrani KS, Bakheit AM. A study of the gait characteristics of patients with chronic osteoarthritis of the knee. Disabil Rehabil 2002; 24:275-80.

42. Gok H, Ergin S, Yavuzer G. Kinetic and kinematic characteristics of gait in patients with medial knee arthrosis. Acta Orthop Scand 2002; 73:647-52.

43. Field A. Discovering statistics using SPSS (3rd ed.). London: Sage Publications; 2009.

44. Bowerman BL and O'Connell RT. Linear statistical models: An applied approach (2nd ed.). Belmont, CA: Duxbury; 1990.

45. Hensor, E, Dube,B, Kingsbury, SR, Tennat, A, Conaghan, PG. Toward a clinical definition of early osteoarthritis: onset of patient-reported knee pain begins on stairs. Data from the osteoarthritis initiative. Arthritis Care \& Res, Vol. 67, No.1, 2015, pp. 40-47.

46. Richards C, Higginson JS. Knee contact force in subjects with symmetrical OA grades: Differences between OA severities. J Biomech 2010; 43:2595-2600.

47. Walter JP, D'Lima DD, Colwell CW, Jr., Fregly BJ. Decreased knee adduction moment does not guarantee decreased medial contact force during gait. J Orthop Res 2010; 28(10):1348-1354. 